\title{
Atom Probe Tomography Characterization of a White Etching Area in a Bearing Steel
}

JeeHyun Kang ${ }^{1}$, Ceri Williams ${ }^{3}$, Babak Hosseinkhani ${ }^{2}$, Pedro E.J. Rivera Díaz del Castillo ${ }^{1}$, Paul A. J. Bagot $^{3}$ and Michael P. Moody ${ }^{3}$

1. SKF UTC, Department of Materials Science and Metallurgy, University of Cambridge, United Kingdom.

2. Department of Lubrication and Metallic Materials, SKF Engineering and Research Centre, The Netherlands.

3. Department of Materials, University of Oxford, United Kingdom.

A white etching area (WEA) is a region of microstructural damage that can form during the operation of martensitic bearing steels [1]. The name arises from their distinctive contrast to the pre-existing structure of these regions when viewed under an optical microscope. The cracking associated with these features is a cause of rolling contact fatigue. It has previously been reported that the WEA exhibit a nanocrystalline structure free of carbides. However, the original microstructure contains a significant amount of carbides in a martensite matrix. This suggests that carbide particles are dissolved due to the high degree of deformation during service. The resulting nanostructure is similar to that observed during a severe plastic deformation process. However, the mechanism for the formation of WEA has not yet been established.

In this study, a white etching area in a martensitic bearing steel has been investigated via atom probe tomography (APT) in order to characterize the distribution of alloying elements. Focused ion beam techniques were used to create site-specific APT specimens incorporating regions of a WEA adjacent to a crack. Nano-sized cell structures are readily visualized in the APT reconstructions, due to the segregation of carbon and silicon to their boundaries, as shown in Figure 1. The cells can be classified into two types by analysis of their size and morphology, as well as enveloping alloying elements. Small carbon clusters which may represent the remains of carbide dissolution have also been observed within a cell. Based upon the experimental results, it is conjectured that carbon and silicon are the key elements controlling the formation of such structure.

\section{References:}

[1] J. Kang et al., Materials Science and Technology, 28, (2102) p. 44. 


\section{Carbon Distribution}

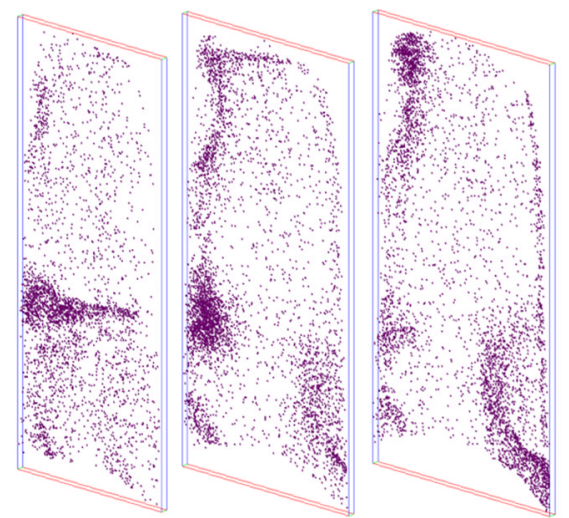

Silicon Distribution
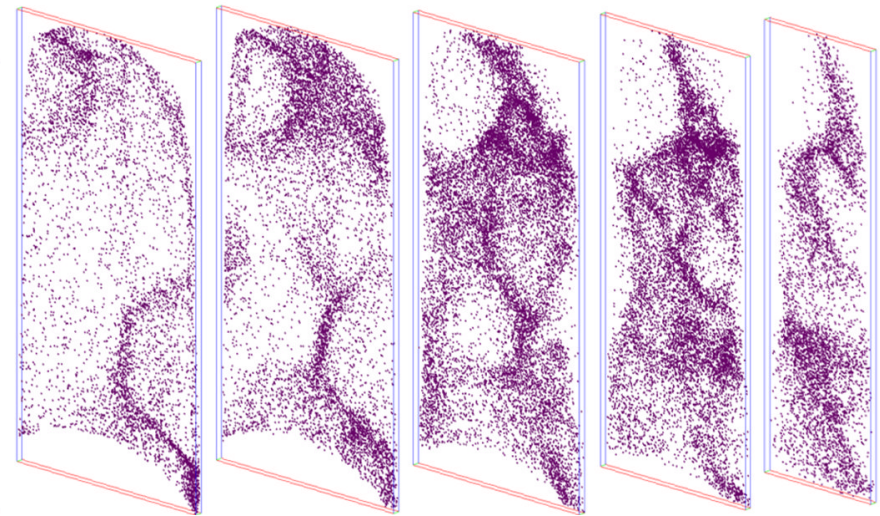

$50 \mathrm{~nm}$
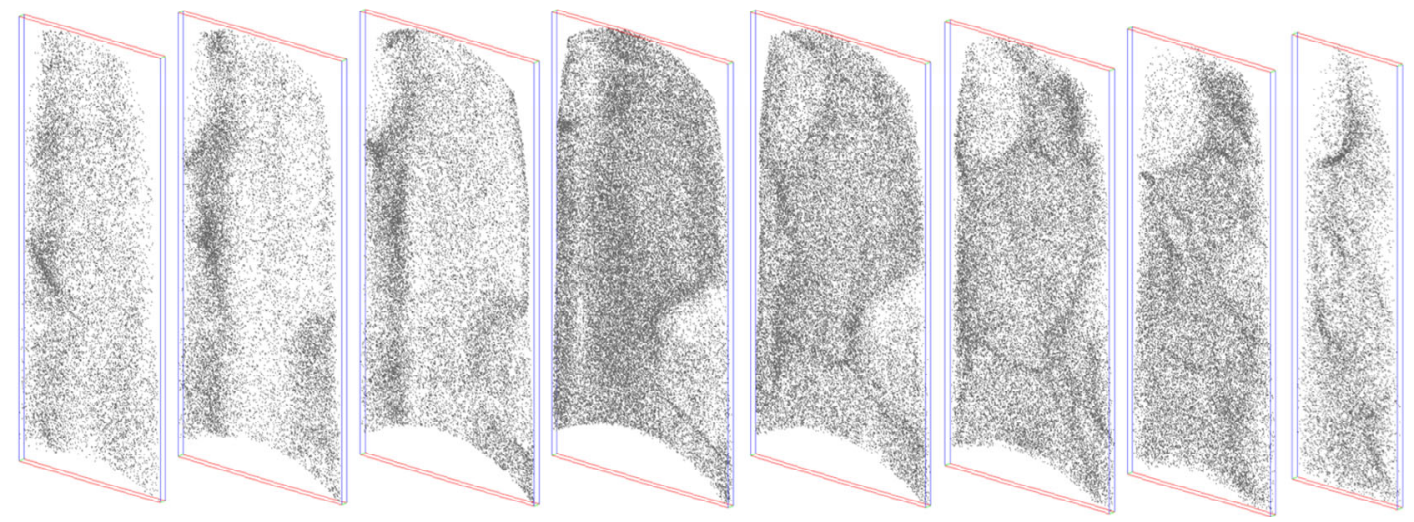

Figure 1. $10 \mathrm{~nm}$ thick slices through the APT reconstruction of a WEA in a bearing steel. Top figure shows distribution of carbon atoms within each slice. Lower figure shows distribution of silicon atoms in corresponding slices. 